\title{
Gonococcal Tonsillar Infections
}

\author{
ANNE BRO-J $\varnothing$ RGENSEN, TAGE JENSEN
}

British Medical fournal, 1971, 4, 660-661

\section{Summary}

Neisseria gonorrhoeae were cultured from tonsillar swabs in six men and six women out of 161 consecutive, unselected, Danish patients (95 men and 66 women) suffering from urogenital or rectal gonorrhoea. Gonococci were found in the tonsils in only one out of 49 foreign men with gonorrhoea. Eleven of the Danish patients admitted orogenital contact at their most recent intercourse. The gonococcal complement fixation test was negative in all except two cases. Standard singledose treatment cured the urogenital and rectal infections promptly but failed to cure the tonsillar infection in five cases, and in recalcitrant cases the organisms were demonstrable for some months.

\section{Introduction}

Gonococcal infection of the oral mucosa is supposed to be rare; most reports are single cases presenting as stomatitis (Schmidt et al., 1961), pharyngitis (Fiumara et al., 1967; Metzger, 1970), ulceration of the tongue (Cowan, 1969), or tonsillitis (Iqbal, 1971). The bacteriological diagnosis was established in only some of these cases (Fiumara et al., 1967; Metzger, 1970; Cowan, 1969). Thatcher et al. (1969) cultured gonococci from the pharynx in one case out of 505 healthy soldiers examined, and Hellgren (1971), in a series of 200 cases of gonorrhoea, found gonococci in the tonsils of one man out of 30 and one woman out of 20 who had had orogenital contact during their most recent intercourse.

In view of the steady increase in gonorrhoea and because orogenital contact is widely practised among Danish patients attending venereal disease clinics in Copenhagen we thought it important to make a more comprehensive study of the frequency of oral gonococcal infections.

\section{Patients and Methods}

The patients comprised 144 men and 66 women suffering from gonorrhoea and 186 men and 77 women not suffering from the disease seen in a public clinic for venereal diseases. Swabs were taken from the urethra in men and also from the rectum in homosexuals. In women swabs were taken from the urethra, cervix, and rectum. Swabs were taken from the tonsils of every patient at their first visit. The tonsils were chosen because they were thought to be the most probable site for gonococci entering the oral cavity to colonize.

All swabs were cultured on the Thayer-Martin selective medium. The immunofluorescence method was used to identify gonococci from the urethra, cervix, and rectum. The sugar fermentation tests were used for cultures from the tonsils, since the presence of Neisseria meningitidis could be a source of error when using immunofluorescence in examining tonsillar swabs. The cultures were examined at the Neisseria Department, State Serum Institute.

Municipal Clinic for Venereal Diseases, Tietgensgade 31, Copenhagen ANNE BRO-J $\varnothing$ RGENSEN, M.D., Registrar TAGE JENSEN, M.D., Consultant

\section{Results}

$N$. gonorrhoeae were found in cultures from the tonsils of 6 men $(6 \%)$ and 6 women (9\%) among 161 consecutive Danish patients ( 95 men and 66 women) suffering from uncomplicated gonorrhoea. Gonococci were cultured from the tonsils of only one among 49 men from other countries, mainly in eastern Europe and the Middle East, treated in the clinic for gonorrhoea. Tonsillar cultures were negative in all the 186 men and 77 women patients not suffering from gonorrhoea.

Ten patients (five men and five women) with tonsillar infection had no localizing signs or symptoms. One developed a febrile tonsillitis five days after treatment. Two had had tonsillectomy during childhood. Three patients had mild to moderate signs of tonsillitis consisting of enlarged tonsils with small yellowwhite exudates but not accompanied by regional lymphadenopathy or fever-one of them complained spontaneously of a sore throat starting four days after practising cunnilingus and the other two had slight discomfort on swallowing. One of these two developed migratory arthralgias and a positive gonococcal complement fixation test at a low titre during the follow-up period, when the tonsils still harboured gonococci but the extratonsillar gonorrhoeal infection had been cured.

\section{FREQUENCY OF OROGENITAL PRACTICES}

Only during the later stages of the investigation were patients consistently questioned about orogenital sexual practices, and therefore answers were obtained from only 71 men and 62 women.

Fifty-seven $(80 \%)$ of the men and $38(61 \%)$ of the women admitted to this form of sexual behaviour, though eight of each practised it only rarely. Thirty $(42 \%)$ of the men admitted cunnilingus and $21(34 \%)$ of the women admitted fellatio with their suspected source of infection. It was often stated that orogenital contact was made only with the regular consort and not with casual acquaintances.

\section{COURSE OF ILLNESS}

All patients were initially given our standard single-dose therapy of ampicillin $2 \mathrm{~g}$ and probenecid $1 \mathrm{~g}$ by mouth. In seven patients three consecutive negative cultures from the tonsils were obtained after the initial treatment. The tonsillar infection was asymptomatic in all these cases. One woman developed a febrile tonsillitis five days after she had received the initial treatment and gonococci had been cultured from the tonsils. A general practitioner treated her with penicillin for one week, and later cultures from the tonsils were negative.

In the remaining five cases gonococci were again cultured from the tonsils after the initial treatment. Two of them had no signs of tonsillitis, while three had symptoms as already described. In one case three negative cultures were obtained after further treatment with a single intramuscular injection of penicillin G 5 megaunits combined with probenecid $1 \mathrm{~g}$. Three patients had further treatment with tetracycline 250 $\mathrm{mg}$ by mouth four times daily for one week. After this treatment cultures from the tonsils were negative in one patient and gonococci reappeared in the other two. In one of these the three preceding cultures had been negative, and it was thought that reinfection was improbable. In these two cases the sensitivity of the gonococci to both penicillin and tetracycline was 
decreased. The strains isolated in the other 11 patients were fully sensitive to these antibiotics.

In the recalcitrant cases, some of which were symptomless, gonococci were harboured in the tonsils for up to eight weeks. $\mathrm{Re}$-exposure to orogenital contact was denied in each case.

\section{Discussion}

Eleven of the 13 patients suffering from tonsillar gonorrhoea had practised orogenital contact at their most recent intercourse, so it is most probable that the infection was acquired in this way. The two exceptions were a heterosexual Mohammedan who denied cunnilingus and a woman who claimed never to practise orogenital contact. Other modes of transferring gonococci to the tonsils must therefore be considered.

In none of our cases was there any indication that the infection had been transferred by kissing. The tonsillar infection was always associated with genital gonorrhoea. However, patients who may have contracted an isolated tonsillar infection, perhaps innocently, are unlikely to consult a venereologist. The throat symptoms when present in our cases were no different from those expected in common nasopharyngeal infections, but their persistence and the fact that cultures remained positive indicate that in our cases they were probably attributable to the gonococci. That gonococcal colonization of the tonsils was most often asymptomatic is suggestive of a carrier state.

Tonsillar gonococcal infections seem to be extremely recalcitrant to treatment. Our routine single-dose treatment failed in about half the cases, whereas it has proved almost completely successful in curing uncomplicated gonorrhoea in both sexes (Bro-Jørgensen and Jensen, 1971). Tetracycline was also ineffectual, and though other regimens have been tried we have not as yet found a reliable therapy. An added difficulty in diagnosis and in assessing the efficacy of treatment is the unreliability of a single negative culture finding. We may have missed some cases on initial examination on account of this.

The high incidence of gonococcal tonsillar infections, their resistance to treatment, the lack of any characteristic signs and symptoms, and the possibility of innocent acquirement of the disease by kissing are factors which presumably may result in a rather high prevalence of this hitherto disregarded gonococcal infection. We suggest that tonsillar gonorrhoea may account for some obscure septicaemic and allergic manifestations as well as for some of the so-called "false-positive" gonococcal complement fixation tests.

\section{References}

Bro-Jgrgensen, A., and Jensen, T. (1971). British fournal of Venereal Diseases, 47. In press.

Cowan, L. (1969). British fournal of Venereal Diseases, 45, 228 Cowan, L. (1969). British fournal of Venereal Diseases, 45, 228.

of Medicine, 276, 1248.
Hellgren, L. (1971). Läkartidnirgen, 68, 569.

Iqbal, Y. (1971). British fournal of Venereal Diseases, 47, 144.

Metzger, A. L. (1970). Annals of Internal Medicine, 73, 267.

Schmidt, H., Hjprting-Hansen, E., and Philipsen, H. P. (1961). Acta Dermato-Venereologica, 41, 324.

Thatcher, R. W., McCraney, W. T., Kellogg, D. S., and Whaley, W. H. (1969). Fournal of the American Medical Association, 210, 315.

\section{MEDICAL MEMORANDA}

\section{Polyuric Acute Renal Failure after Methoxyflurane and Tetracycline}

\section{E. A. PROCTOR, F. L. BARTON}

\section{British Medical fournal, 1971, 4, 661-662}

Methoxyflurane has been in use as an anaesthetic agent for over a decade. Initial studies on this drug failed to show any hepatic or renal toxicity. There have, however, been several reports since 1964 associating methoxyflurane anaesthesia with a postoperative raised urine output and azotaemia. Tetracycline drugs have been reported to produce a similar picture and it has been suggested that the combination of methoxyflurane anaesthesia with tetracycline administration is more likely to result in this clinical syndrome (Kuzucu, 1970). We present such a case and discuss some of the unusual features.

\section{Case Report}

A previously healthy 33-year-old man was admitted to the intensive care unit with severe chest injuries after a car accident. He was shocked, had a four-rib flail segment of the left mid-chest anteriorly, fractures of his second, third, and fourth ribs on the

Leeds General Infirmary, Leeds LS1 3EX

E. A. PROCTOR, M.B., B.S., Registrar, Renal Research Unit

F. L. BARTON, F.F.A., R.C.S., Registrar, Department of Anaesthesia (Present address : Brompton Hospital, London SW 3 ) right, a right-sided tension pneuomothorax, and a progressively widening mediastinum. This latter feature was a haemomediastinum due to an avulsed right subclavian artery, which was repaired under heart-lung bypass. The surgical management of this case is dealt with more fully in a report by Girdwood et al. (1972).

Postoperatively he made good progress; his cardiac output and renal function were good. His early postoperative management and therapy consisted of intermittent positive-pressure ventilation to control the flail segment, blood and fluids as necessary, regular bronchial toilet, and che si physiotherapy four times daily, with intravenous fentanyl $0.1 \mathrm{mg}$ for analgesia. In addition the following drugs were given: ampicillin $250 \mathrm{mg}$ plus cloxacillin $250 \mathrm{mg}$ intramuscularly four times daily and streptomycin $500 \mathrm{mg}$ intramuscularly twice daily for six days, ascorbic acid $500 \mathrm{mg}$ intramuscularly twice daily (later $400 \mathrm{mg}$ by mouth), and diazepam $5 \mathrm{mg}$ intramuscularly or papaveretum $5 \mathrm{mg}$ intramuscularly or both as required for analgesia and sedation.

From the seventh postoperative day, because of the large amounts of papaveretum and fentanyl that he was receiving, chest physiotherapy was carried out three times daily under methoxyflurane analgesia. Methoxyflurane $(0 \cdot 1-3 \%)$ was delivered together with $50 \%$ nitrous oxide in oxygen via a Pentec vaporizer on a Boyle's anaesthesia machine using a 10-1. fresh gas flow and a Mapleson C circuit for about 20 minutes each time. On the same day, because Klebsiella aerogenes was isolated from his bronchial secretions, the antibiotics were changed to tetracycline $100 \mathrm{mg}$ intramuscularly four times daily after a loading dose of $500 \mathrm{mg}$, plus streptomycin $0.5 \mathrm{~g}$ twice daily. Then, because of the appearance of Pseudomonas pyocyanea in his secretions, antibiotics were stopped after the 10th postoperative day in the hope of avoiding further superinfection after the antibiotic suppression of normal flora.

His general condition continued to improve until the 13th postoperative day, when he was noticed to be dehydrated. He improved both clinically and biochemically with rehydration. $\mathrm{He}$ was then maintained with a fluid intake of 4.51 ./day, but within three days he was again dehydrated and we realized that he was suffering from an iatrogenic diabetes insipidus. He was not hyper- 